\title{
A Particle Filter for Ground Obstacle Tracking in the Airfield
}

\author{
Kevin Theuma ${ }^{1}$, David Zammit-Mangion ${ }^{2}$, Jason Gauci ${ }^{3}$ and Kenneth Chircop ${ }^{4}$ \\ University of Malta, Msida MSD 2080, Malta \\ and \\ Nicolas Riviere ${ }^{5}$ \\ ONERA, Toulouse, 31055, France
}

\begin{abstract}
This paper addresses pilot error due to distractions, lack of situational awareness and misjudgment of separation during taxi manoeuvers. A display is being developed to minimize these errors and assist pilots to determine safe passage while navigating during taxi. Part of the development consisted of the implementation of a tracking algorithm - based on the particle filter - which is being presented in this paper. The tracking algorithm tracks obstacles that have been detected and reconstructed using stereo vision techniques. Afterwards, the tracked obstacles are highlighted on a display. Experiments show that the algorithm successfully detects and tracks aircraft in an airfield.
\end{abstract}

\section{Introduction}

Unlike other phases of flight, such as cruise and landing, taxi is still carried out manually by pilots. During taxi operations, pilots navigate aircraft manually based on their judgement and instructions from Air Traffic Control. Therefore, these operations are prone to pilot error due to distractions, lack of situational awareness and misjudgment of separation [1]. In fact, recently, there were various reports of aircraft involved in collisions with buildings, vehicles and other aircraft [2] [3] [4]. In order to avoid such errors from occurring, industry and academia have been researching technologies for assisting pilots during taxi. For example, a 7th Framework Programme project under the name of "All Condition Operations and Innovative Cockpit Infrastructure" involved multiple institutions working on a solution for enhancing situational awareness and improving aircraft safety during this phase of flight [5]. Apart from improving safety, research in this area is also concerned with improving time efficiency and enabling all weather operations [6]. The latter can help reduce and possibly eliminate delays that usually occur in poor visibility conditions.

This paper presents a solution that can accurately track obstacles which are in turn presented to pilots on a topview display. Such a tool would assist pilots by raising awareness of nearby obstacles and aiding them in judging distances of separation between the aircraft and nearby obstacles to determine safe passage. Obstacles can be detected by different sensors such as electro-optical, infrared, LIDAR and RADAR sensors. Gauci et. al. [7] detected obstacles with a pair of electro-optical sensors through stereo vision. However, results demonstrate that as the range increases so do the measurement errors. Shi et. al. [8] applied stereo vision techniques to infrared sensors but measurement errors still increase with range. LIDAR sensors, on the other hand, are more accurate but have a limited vertical field of view which means that nearby obstacles, depending on their vertical position, can remain undetected. RADAR sensors can be either active or passive. Active RADAR sensors interfere with other sensors of the same type, meaning that they can interfere with the Surface Movement Radar. Meanwhile, passive RADAR sensors are susceptible to multipath effects. Due to these limitations, one sensor alone is not sufficient for reliable and accurate detection of obstacles in the airfield. Therefore, a multi-sensor system is being proposed. In fact, the work being presented in this paper is intended to be used as part of a multi-sensor system consisting of a pair of electro-optical cameras and a LIDAR sensor.

\footnotetext{
${ }^{1}$ Ph.D. student, Institute of Aerospace Technologies, and AIAA Student Member

${ }^{2}$ Director, Institute of Aerospace Technologies, and AIAA Senior Member

${ }^{3}$ Lecturer, Institute of Aerospace Technologies, and AIAA Member

${ }^{4}$ Visiting Lecturer, Institute of Aerospace Technologies, and AIAA Member

${ }^{5}$ Senior Research Engineer, Optronics Department
} 
Previous work [9] took advantage of two electro-optical sensors to detect and reconstruct obstacles in 3D space. Detected obstacles were then presented to pilots on a top-view display. However, the algorithms and experiments were limited to static scenes and obstacles. This paper builds upon that work by proposing a tracking algorithm based on the Particle Filter [10] which can be used to track dynamic obstacles. This algorithm allows for the full reconstruction of obstacles when viewed from different viewpoints.

The paper is organized as follows: Section 2 explains the developed algorithm in detail, Section 3 describes the design of experiment, Section 4 presents and discusses the results, and Section 5 concludes this paper.

\section{Algorithm for Obstacle Detection, Tracking and Visualization}

\section{A. Overview of the Algorithm}

The developed algorithm detects and tracks obstacles by processing a collection of points in 3D space referred to as a point cloud. These points can be acquired by reconstructing scenes through stereo vision techniques or by using LIDAR sensors. LIDAR sensors give a more accurate point cloud representation than stereo vision techniques; however, in this work, 3D reconstruction is carried out using stereo vision techniques. The reason for this approach is that, if the detection and tracking algorithms work with point clouds reconstructed through stereo vision, they are expected to work with ones obtained by LIDAR sensors as well.

Stereo vision processes multiple images in order to obtain a 3D representation of the scene. These images can be captured by a single camera over a duration of time or by multiple cameras at a single time instance. For reconstruction with the use of a single camera, the camera must be in motion. Meanwhile, reconstruction with the use of multiple cameras will work regardless of whether the cameras are in motion or not. In this application, obstacles have to be detected even when the aircraft is parked or stopped. Therefore, two cameras are used.

Scenes are reconstructed in 3D space by processing images captured by these two cameras through the use of stereo vision techniques. Afterwards, they are filtered, processed and analyzed in order to detect obstacles. Following detection, obstacles are associated with information from previous measurements and are then tracked. Tracking algorithms process a sequence of measurements to filter noise and increase confidence. The resulting estimates are used to update the positions of the obstacles. Finally, the obstacles are mapped onto a top-view image and their points are encapsulated by polygons. These polygons highlight hazardous regions that are occupied by obstacles. A block diagram of the algorithm is illustrated in Fig. 1.

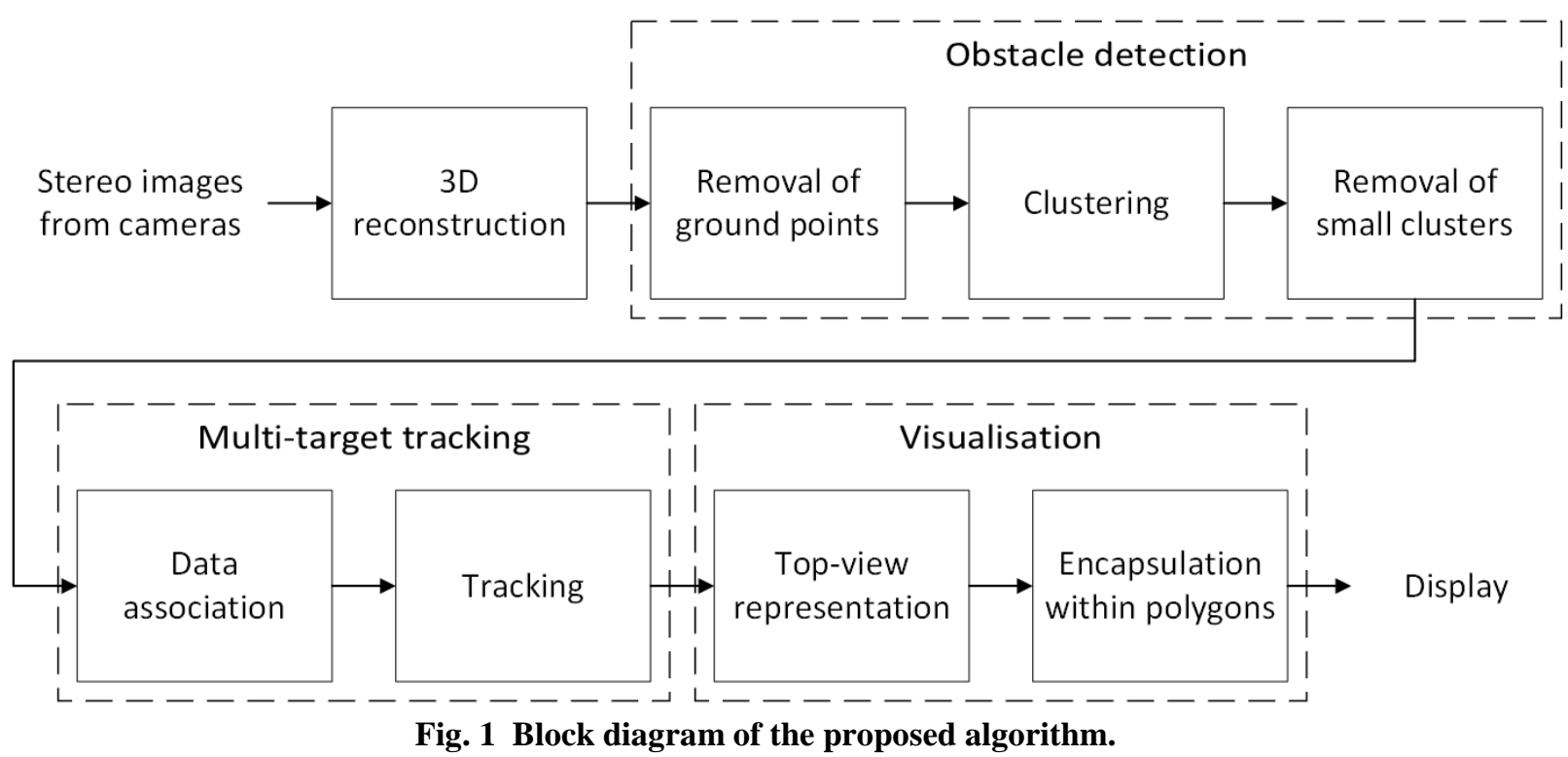

\section{B. 3D Reconstruction}

When objects are captured by cameras from different viewpoints, they appear to be shifted. If two cameras are aligned such that they are facing the same direction and are only separated laterally, the lateral shift (referred to as the 
disparity) is inversely proportional to the range. Hence, this relationship can be used to recover range information for each pixel within the image by noting the disparities.

Stereo vision algorithms consist of three main steps which are Rectification, Correspondence and Triangulation. The Rectification step corrects distortions in the captured stereo images and aligns the optical axes of the stereo cameras i.e. as if the stereo cameras were parallel to each other. The Correspondence step looks for corresponding pixels between the left and right images and computes the disparities. The Triangulation step maps pixels into 3D space based on their disparities.

Lenses can cause two types of distortions which are radial and tangential distortions. These distortions can be characterized by a set of coefficients which are obtained through calibration. Increasing the number of coefficients allows for describing more complex distortions. However, during calibration, this may eventually result in a phenomenon known as overfitting which can worsen the correction and instead produce other distortions. To avoid this, the tangential distortion is assumed to be zero and the radial distortion is characterized by two coefficients. In the Rectification step, distortion is corrected by mapping the images according to Eqs. 1 and 2:

$$
\begin{aligned}
& x_{2}=x_{1}\left(1+k_{1} r^{2}+k_{2} r^{4}\right) \\
& y_{2}=y_{1}\left(1+k_{1} r^{2}+k_{2} r^{4}\right)
\end{aligned}
$$

where $\left(x_{1}, y_{1}\right)$ is the pixel coordinate in the undistorted image relative to the principal point, $\left(x_{2}, y_{2}\right)$ is the pixel coordinate in the distorted image relative to the principal point, $r$ is distance from the principal point, and $k_{1}$ and $k_{2}$ are the radial distortion coefficients.

Distortion correction is followed by alignment of the two views which is performed through a series of translations and rotations. The translations and rotations between the two cameras are found through calibration and are known as the extrinsic camera parameters.

Once the images are rectified, the algorithm proceeds with the Correspondence step which computes the disparities for all pixels. For this step, the Semi Global Block Matching (SGBM) method is used. SGBM is based on Semi Global Matching (SGM) [11] but matches blocks of pixels rather than individual pixels. The matching is based on an energy cost function which assigns a score for each potential disparity. The energy cost function includes two penalty terms $\mathrm{P}_{1}$ and $\mathrm{P}_{2}$. These are used to filter noise in the estimated disparities while preserving discontinuities. $\mathrm{P}_{1}$ penalizes small disparity changes (less than or equal to one pixel) and $\mathrm{P}_{2}$ penalizes large disparity changes (greater than one pixel). Once the energy costs are calculated, they are aggregated along different directions as shown in Fig. 2. Finally, the disparities with the lowest energy costs are selected.

\section{Paths from all Directions $r$}

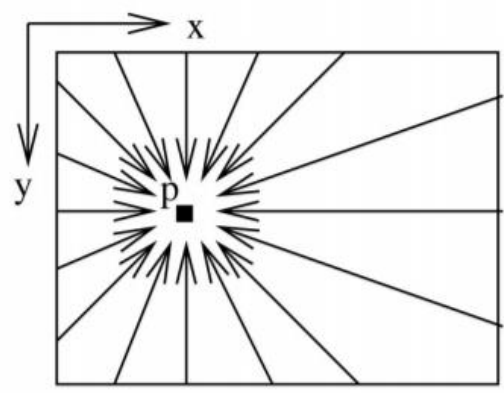

Fig. 2 Directions of cost aggregation per pixel [11].

In the proposed implementation, a block size of $9 \times 9$ pixels is used. $P_{1}$ is set to 1944 and $P_{2}$ is set to 7776 . To increase the Signal to Noise Ratio (SNR) in the computed disparities, for each pixel, the disparity with the lowest energy cost (i.e. the selected disparity) and the disparity with the second lowest energy costs are compared. If the difference in energy costs is less than $15 \%$, then the selected disparity is considered to be invalid. Additionally, to increase the SNR of the computed disparities even further, disparities are computed for both left and right camera images thus producing two disparity maps. It is ensured that corresponding disparities are consistent with each other 
by checking that the difference in disparities does not exceed one pixel. Inconsistent disparities are considered to be invalid.

Following the Correspondences step, the Triangulation step reconstructs pixels in 3D space based on their disparities. Prior to reconstruction, the disparity image is filtered to remove distant points and small regions of discontinuous disparities. Distant points are removed by thresholding disparities by the disparity that corresponds to a longitudinal distance of $100 \mathrm{~m}$. Disparities at or above the threshold are left intact while the others are discarded. Small regions of discontinuous disparities are filtered by first clustering neighboring pixels whose disparities differ by at most two pixels and then discarding disparities within clusters of less than 200 pixels. After filtering the disparity image, the images are reconstructed in 3D space by projecting pixels according to Eqs. 3, 4 and 5 [12].

$$
\begin{gathered}
X=\frac{b\left(x-c_{x}\right)}{D} \\
Y=\frac{b\left(y-c_{y}\right)}{D} \\
Z=\frac{b f}{D}
\end{gathered}
$$

where $X, Y$ and $Z$ are the lateral, vertical and longitudinal positions relative to the left camera respectively, $b$ is the baseline distance i.e. the lateral distance between the two cameras, $f$ is the focal length of the cameras, $D$ is the disparity, $x$ and $y$ are the horizontal and vertical positions of the pixels within the disparity image respectively, and $c_{x}$ and $c_{y}$ are the horizontal and vertical coordinates of the principal point respectively.

\section{Obstacle Detection}

Once the image pixels are projected to 3D space, obstacles can be detected by processing and analyzing the reconstructed point cloud. Up to this point, the point cloud consists of points belonging to obstacles and to the ground (assuming that all points above the ground are obstacles). Assuming that the ground is planar and orthogonal to the sensors, ground points are filtered by removing points whose vertical position is below a pre-defined threshold. This threshold corresponds to $1 \mathrm{~m}$ above ground to compensate for noise in the reconstructed point cloud.

The remaining points may belong to multiple obstacles. To distinguish between obstacles, points that are within a distance of $2 \mathrm{~m}$ from each other are clustered together and each cluster is considered to belong to a separate obstacle. Small clusters which span less than $2 \mathrm{~m}$ in all of the three dimensions (i.e. longitudinally, laterally and vertically) are discarded because these tend to be a result of noise. The remaining clusters are the detected obstacles which are then tracked by the tracking algorithm described in Subsection E.

\section{Methods for Multi-Target Tracking in 3D Space}

The detected obstacles are defined by a cluster of points. Tracking clusters of points is a complex task due to the large amount of points usually present and their non-uniqueness which makes data association of corresponding points captured in successive time steps a non-trivial problem. This can be circumvented by representing obstacles in other formats such as bounding boxes.

The various representations for tracking obstacles in 3D space can be split into four main categories. They consist of:

1) Methods that encapsulate obstacles within a bounding box and then track the bounding box vertices,

2) Methods that compute the centroids of the obstacles and then track the centroids,

3) Methods that identify points of interest, better known as key-points, and then track these points, and

4) Methods that track the point cloud as a whole.

The first method finds the minimum and maximum coordinates of the obstacle points in each dimension. The resulting coordinates correspond to the vertices of a box bounding the obstacle which are then tracked with the tracking algorithm [13]. However, the shape of the bounding box is inadequate at characterizing complex structures such as aircraft and therefore, the vertices of the bounding box might not be consistent with subsequent measurements.

The second method, as proposed by Schutz et. al. [14], builds an occupancy grid for each obstacle and then computes and tracks the centroids of the occupancy grids. To build the occupancy grid, the volume occupied by the 
obstacle points is divided into equally sized cells and the probability that a cell is occupied is calculated based on the obstacle points within the cell. The advantage of this approach is that it accumulates data over time to eventually reconstruct the whole obstacle. However, since points are captured from one view at a time, the centroid shifts while building the occupancy grid.

The third method consists of selecting key-points from the obstacle points, computing corresponding descriptors, associating key-points in subsequent measurements based on their descriptors and tracking them [15]. Tests using this method showed that key-points of subsequent measurements are not consistent and hence do not produce satisfactory results.

The fourth method tracks obstacle point clouds as a whole by finding transformations (i.e. translations and rotations) between successive measurements and then filtering them. The implementation by Ueda [16] tracks a predefined point cloud whose structure remains static during execution time. The work being presented in this paper extends this idea further by accurately registering and accumulating points to progressively reconstruct the obstacle from different viewpoints over time.

\section{E. Proposed Multi-Target Tracking Algorithm}

The detected obstacles are tracked with the Sequential Importance Resampling (SIR) Particle Filter. It corrects their position by estimating an offset in position and orientation. Additionally, the same algorithm is adapted to register and accumulate obstacle points. A particle filter consists of a number of hypotheses, referred to as particles. Each particle is made up of one or more variables called states and a weight (usually equivalent to the probability of the hypothesis being correct).

The implemented particle filter has 100 particles, each consisting of the following nine states: the position offset in 3D coordinates, the angular offset in Euler angles, the lateral and longitudinal velocities and the rate of change of yaw.

When a new obstacle is detected, a new particle filter is initialized by setting all offsets to zero and setting all weights to a uniform value that sums up to 1. This step is formulated as in Eqs. 6 and 7:

$$
\begin{aligned}
& a_{i}=\left.0\right|_{i=1 \ldots N} \\
& w_{i}=\left.\frac{1}{N}\right|_{i=1 \ldots N}
\end{aligned}
$$

where $a_{i}$ is a generalization of all states of particle $i, w_{i}$ is the weight of particle $i$ and $N$ is the number of particles. This particle filter and the obstacle points are linked and saved together. The obstacle point cloud is used to accumulate points whenever the same obstacle is detected while the particle filter is used to compute an offset for the obstacle point cloud to correct its position and orientation.

In subsequent time steps, the created particle filter is updated to correct obstacle positions based on current measurements. The update starts by predicting the offset in position and orientation of the obstacle. This involves sampling particles from a Gaussian distribution where the mean is equal to the previous value of the particle and the standard deviation is a pre-defined value which varies from one state to another. The states are updated as shown in Eqs. 8 to 16 :

$$
\begin{gathered}
X_{i}^{*}[k]=X_{i}^{*}[k-1]+\dot{X}_{i}^{*}[k] t+\left.g(\mu, \sigma)\right|_{i=1 \ldots N} \\
Y_{i}^{*}[k]=Y_{i}^{*}[k-1]+\left.g(\mu, \sigma)\right|_{i=1 \ldots N} \\
Z_{i}^{*}[k]=Z_{i}^{*}[k-1]+\dot{Z}_{i}^{*}[k] t+\left.g(\mu, \sigma)\right|_{i=1 \ldots N} \\
\dot{X}_{i}^{*}[k]=\dot{X}_{i}^{*}[k-1]+\left.g(\mu, \sigma)\right|_{i=1 \ldots N}
\end{gathered}
$$




$$
\begin{gathered}
\dot{Z}_{i}^{*}[k]=\dot{Z}_{i}^{*}[k-1]+\left.g(\mu, \sigma)\right|_{i=1 \ldots N} \\
\alpha_{i}[k]=\alpha_{i}[k-1]+\dot{\alpha}_{i}[k] t+\left.g(\mu, \sigma)\right|_{i=1 \ldots N} \\
\beta_{i}[k]=\beta_{i}[k-1]+\left.g(\mu, \sigma)\right|_{i=1 \ldots N} \\
\gamma_{i}[k]=\gamma_{i}[k-1]+\left.g(\mu, \sigma)\right|_{i=1 \ldots N} \\
\dot{\alpha}_{i}[k]=\dot{\alpha}_{i}[k-1]+\left.g(\mu, \sigma)\right|_{i=1 \ldots N}
\end{gathered}
$$

where $X^{*}$ is the horizontal offset in the $X$-direction, $Y^{*}$ is the vertical offset in the $Y$-direction, $Z^{*}$ is the horizontal offset in the $Z$-direction, $\dot{X}^{*}$ is rate of change of $\dot{X}, \dot{Z}^{*}$ is the rate of change of $\dot{Z}, \alpha$ is the offset in yaw, $\beta$ is the offset in pitch, $\gamma$ is the offset in roll, $\alpha$ is the rate of change of $\alpha, k$ is the time step, and $t$ is the time period.

The updated offsets of each particle are used to transform the cloud accumulating obstacle points into a new point cloud through a translation by $\left(X^{*}, Y^{*}, Z^{*}\right)$ and a rotation by $(\alpha, \beta, \gamma)$. The transformed point cloud is compared to the current measurements by finding the closest points between the two point clouds and calculating the Euclidian distance between them. Pairs of points that are farther than $0.3 \mathrm{~m}$ are considered as outliers and discarded. Meanwhile, the rest are considered as inliers and the mean of their distances is calculated. If the number of inliers is smaller than 1000, the weight of the particle is set to 0 . If it is greater or equal to 1000 , the weight is multiplied by the inverse of the mean distance as shown in Eq. 17:

$$
w_{i}[k]=\left.w_{i}[k-1] *\left(\frac{\sum_{j=0}^{M} d_{j}}{M}\right)^{-1}\right|_{i=1 \ldots N}
$$

where $M$ is the number of point pairs and $d$ is the distance between the pair of points.

The total weight of all particles must sum up to 1 so the weights are normalized as in Eq. 18:

$$
w_{i}^{*}[k]=\left.\frac{w_{i}[k]}{\sum_{j=0}^{N} w_{j}[k]}\right|_{i=1 \ldots N}
$$

where $w^{*}$ is the normalized particle weight.

If the particle weights sum up to 0 , then that means that the obstacle being tracked was not found in the point cloud reconstructed in the current time step. Therefore, the weights are reverted back to their previous values and a counter is incremented to record the number of times that the obstacle was not detected. If an obstacle is not detected for three consecutive time steps, then its track is discarded.

Once the weights are normalized, the following step is to resample the particles. This step is only carried out if one of the weights is zero or if the effective number of particles is less than half the total number of particles. The latter is formulated as in Eqs. 19 and 20:

$$
\begin{gathered}
N_{\text {eff }}<0.5 N \\
N_{\text {eff }}=\frac{1}{\sum_{i=1}^{N}\left(w_{i}^{*}[k]\right)^{2}}
\end{gathered}
$$


where $N_{\text {eff }}$ is the effective number of particles. The particles are resampled by randomly drawing a new set of particles from the current one with probabilities proportional to the particle weights. Afterwards, the old particles are discarded and replaced by the newer ones.

The next step is to compute the corrected state estimates. This is carried out by calculating the weighted sum as shown in Eq. 21:

$$
\bar{a}=\sum_{i=0}^{N} a_{i}[k] w_{i}^{*}[k]
$$

where $\bar{a}$ is a corrected state estimate. The corrected state estimates define the positional and angular offsets of the obstacle point cloud.

The final step is to accumulate points into the obstacle point cloud. This consists of registering the point clouds and then accumulating overlapping clusters. For registration, the algorithm uses the transform given by the particle with the smallest mean distance. The inverse of this transform is calculated and used to register the point cloud currently being observed to the obstacle point cloud linked with the particle filter. The overlapping clusters are merged together and saved into the obstacle point cloud linked with the particle filter. Preliminary tests showed that, over time, as the number of points representing an obstacle increases, the algorithm becomes slower and takes up more memory. Therefore, in the experiments, the obstacle point cloud had to be down-sampled using a voxel grid at each time step.

After updating all particle filters, clusters (in the point cloud being observed) that are not accumulated by any particle filter are considered as new detections and hence a new particle filter is initialized for these clusters.

\section{F. Visualization}

Once the tracking step is complete, the updated obstacle information is presented onto a top-view display. First, obstacle points are translated and rotated according to their track estimates and are then combined into a new point cloud. The vertical coordinates are discarded such that the point cloud results into a top-view image. Then, the points of each obstacle are enclosed within a red polygon by finding their convex hull using the algorithm proposed by Sklansky [17]. The polygons highlight the hazardous regions occupied by obstacles. However, these might not be adequately large to be clearly visible on the display. To circumvent this issue, a buffer region is drawn around small polygons. This is carried out by duplicating the polygons, dilating the duplicated polygons by a circular structuring element having a diameter of nine pixels and then removing the areas occupied by the original polygons from the dilated ones. To determine whether the highlighted area is small, the area of each buffer region is compared to that of the original polygon. If the buffer region occupies an area greater than that of the original polygon, the buffer region is drawn in amber, else it is not drawn.

\section{Design of Experiment}

The experiments were carried out by processing images rendered from a synthetic environment. This synthetic environment consisted of an airfield and two Boeing 777 aircraft which were modelled using Blender [18], a 3D modelling software. One aircraft was parked and hence remained static throughout the simulation while the other aircraft was moving at 20 knots. The purpose of simulating more than one aircraft was to validate that data association works properly. Additionally, the static aircraft tested the effectiveness of the tracking algorithm on static targets while the moving aircraft tested the effectiveness of the tracking algorithm on moving targets.

Two cameras were inserted $1.5 \mathrm{~m}$ apart and $3 \mathrm{~m}$ above the ground to simulate a stereo vision system attached to the nose of an Airbus A320. The cameras had a resolution of $3840 \times 2160$ pixels, a frame rate of $30 \mathrm{~Hz}$, a $20 \mathrm{~mm}$ focal length and a $35 \mathrm{~mm}$ sensor size. They were oriented to face both aircraft at the start of the simulation. Their placement was such that the closest and farthest visible points of the static aircraft were $19 \mathrm{~m}$ and $23 \mathrm{~m}$ away respectively. The closest point of the moving aircraft was $43 \mathrm{~m}$ away while its farthest point was $98 \mathrm{~m}$ away. The simulation was run till the moving aircraft exited the field of view of the cameras i.e. for 6.67 seconds. A render simulating an image captured by the left camera is shown in Fig. 3. 


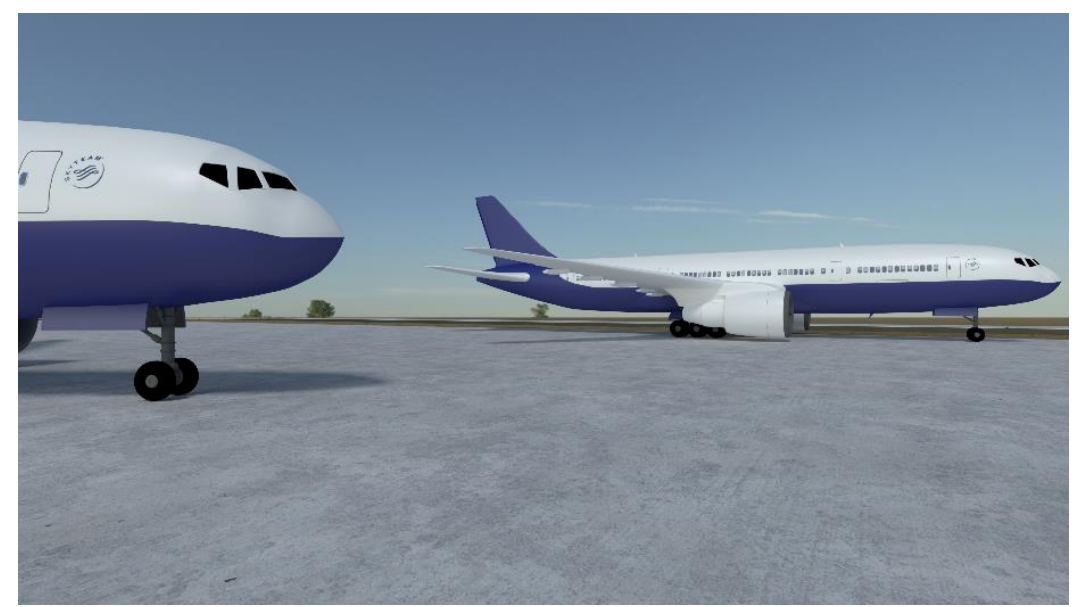

Fig. 3 A render of the airfield created in Blender.

For rendering the synthetic images within Blender, the Cycles engine was used. 200 frames were rendered for the left camera and another 200 frames were rendered for the right camera. These images were processed using the developed algorithm. It was ensured that the visualizations on the display correctly presented detected obstacles. The resulting point clouds were compared to the original 3D meshes within CloudCompare [19] (a software for comparing point clouds) in order to evaluate the performance of the algorithms and to measure their error.

\section{Results}

Throughout the duration of the experiment, detected obstacles were correctly visualized on the developed display. The polygon representing the static aircraft remained fixed, while the polygon representing the moving aircraft gradually moved from left to right and slightly downwards. This matches well with the behavior of the aircraft in the synthetic environment. At the start of the experiment, some parts of the aircraft were represented by separate polygons. This issue was automatically solved as more points were accumulated over time because, through the accumulated point cloud, the algorithm was able to associate different parts to the same obstacle.

Fig. 4 shows the visualization obtained after processing the first frame. The aircraft to which the sensors are attached is illustrated in white. The range circles indicate the distances from the sensors in meters. The closest red polygon represents the static aircraft. Since only a small portion of the aircraft was apparent within the captured images, the polygon only represents part of the aircraft. The other polygons represent the moving aircraft. Due to discontinuities in the point cloud, different parts of the aircraft are detected as separate obstacles and are represented by separate polygons.

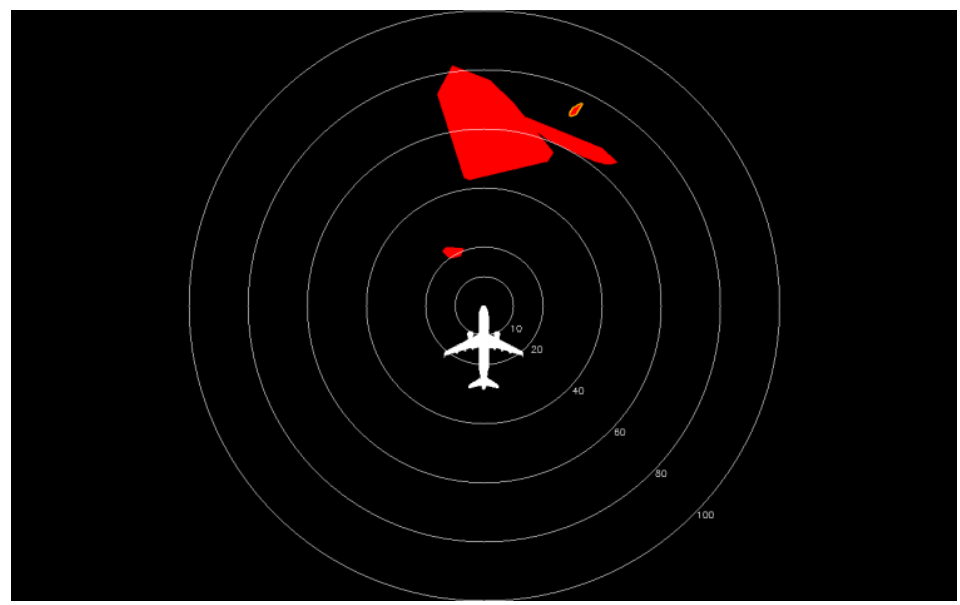

Fig. 4 The visualization resulting from processing the 1st frame.

As can be seen from Fig. 5, which is the result obtained after processing the second frame, once more points were accumulated, different parts of the aircraft became associated with the same obstacle. 


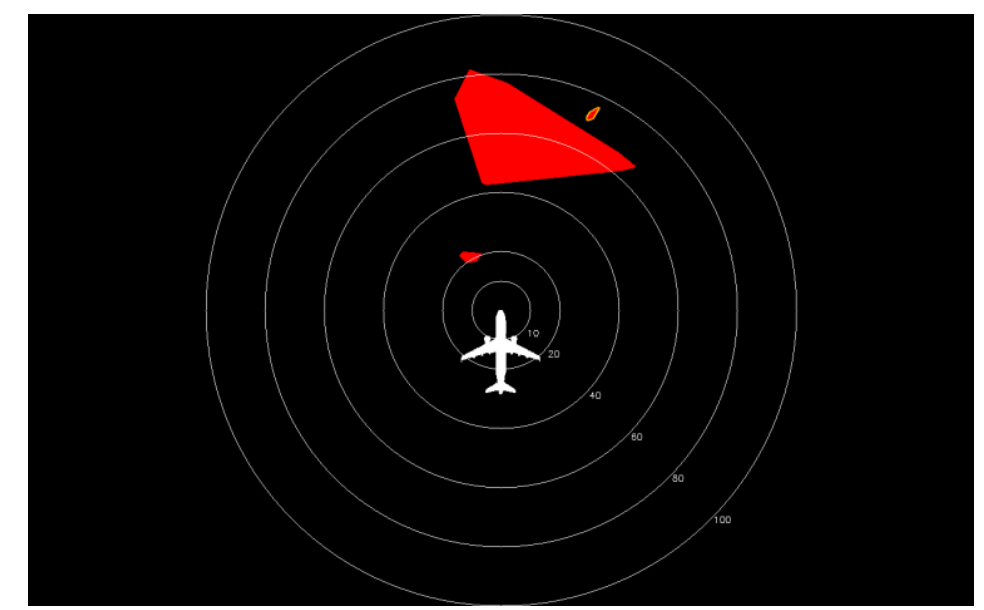

Fig. 5 The visualization resulting from processing the 2 nd frame.

In fact, Fig. 6, which is the result obtained after processing the 180th frame, shows that all parts became associated to the same obstacle. It can be also seen that from the 1st frame to the 180th frame, the polygon representing the moving aircraft moved to the right and slightly downwards.

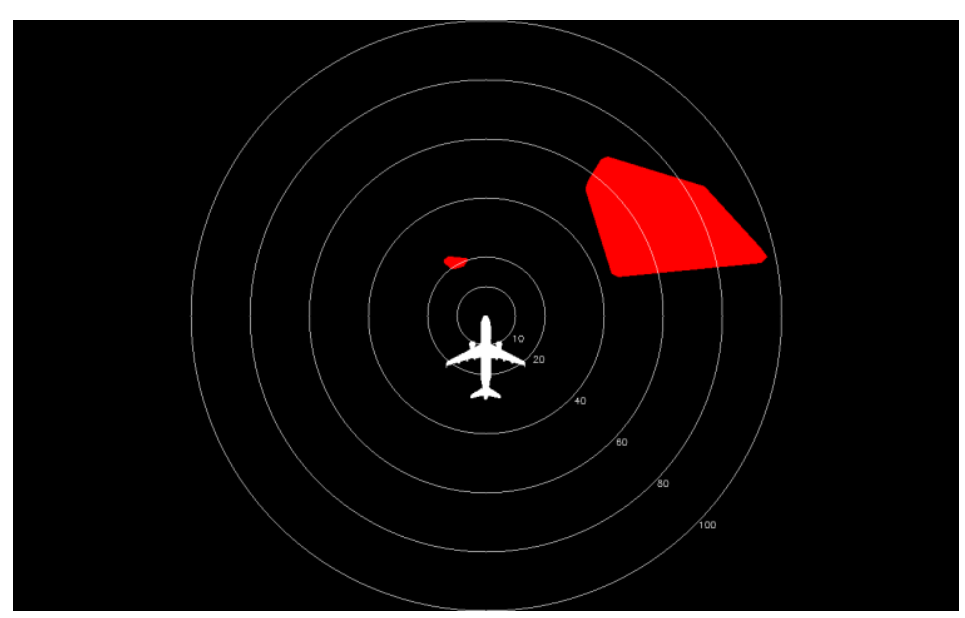

Fig. 6 The visualization resulting from processing the 180th frame.

Fig. 7 shows the result obtained after processing the 200th frame. At this point, the moving aircraft was no longer apparent within the captured images and since the aircraft was not detected for more than 3 consecutive time steps, its track was deleted. 


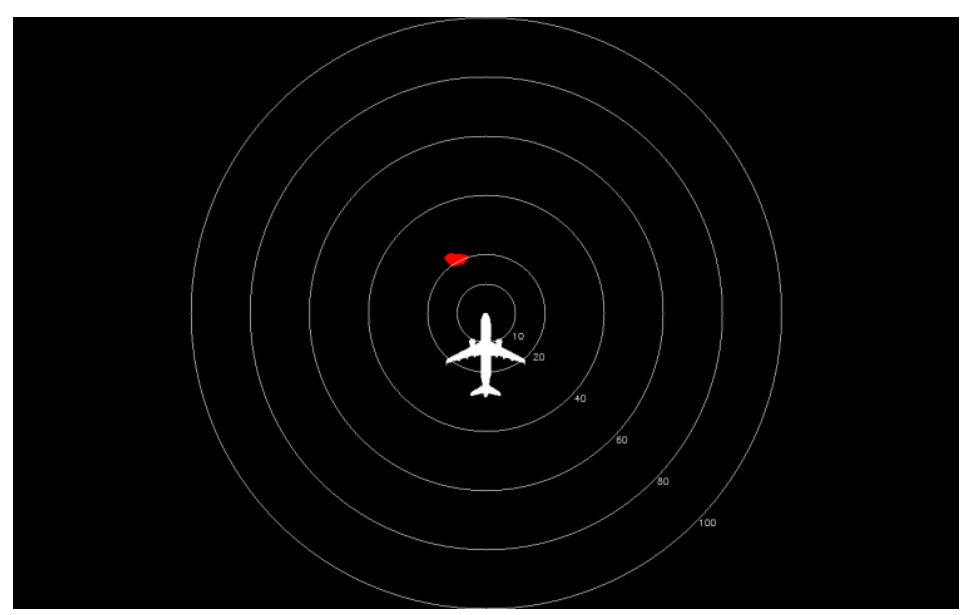

Fig. 7 The visualization resulting from processing the 200th frame.

The mean absolute error and the standard deviation of the obstacle point cloud representing the static obstacle at different time steps are presented in Fig. 8 and Fig. 9 respectively. It can be seen that they vary slightly over time except for some random spikes. Unfortunately, since the points are down-sampled at each time step, their distribution is lost and hence the accuracy does not improve.

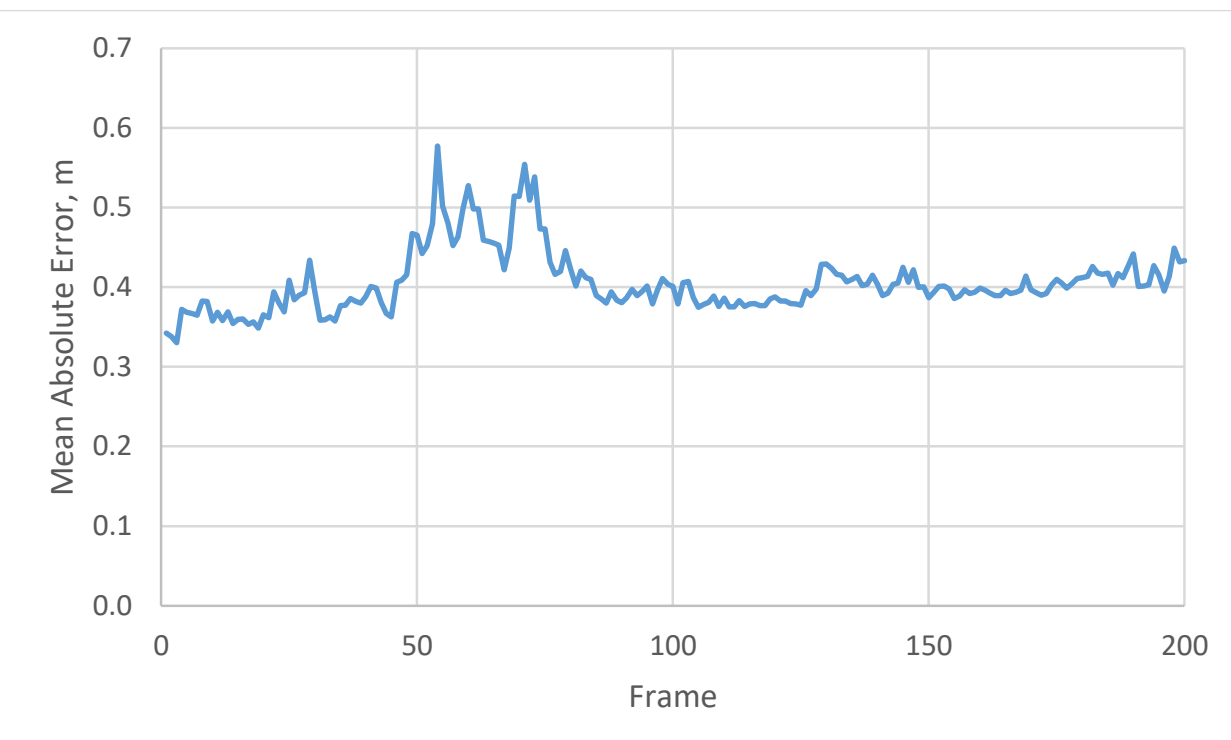

Fig. 8 The mean absolute error of the point cloud representing the static obstacle. 


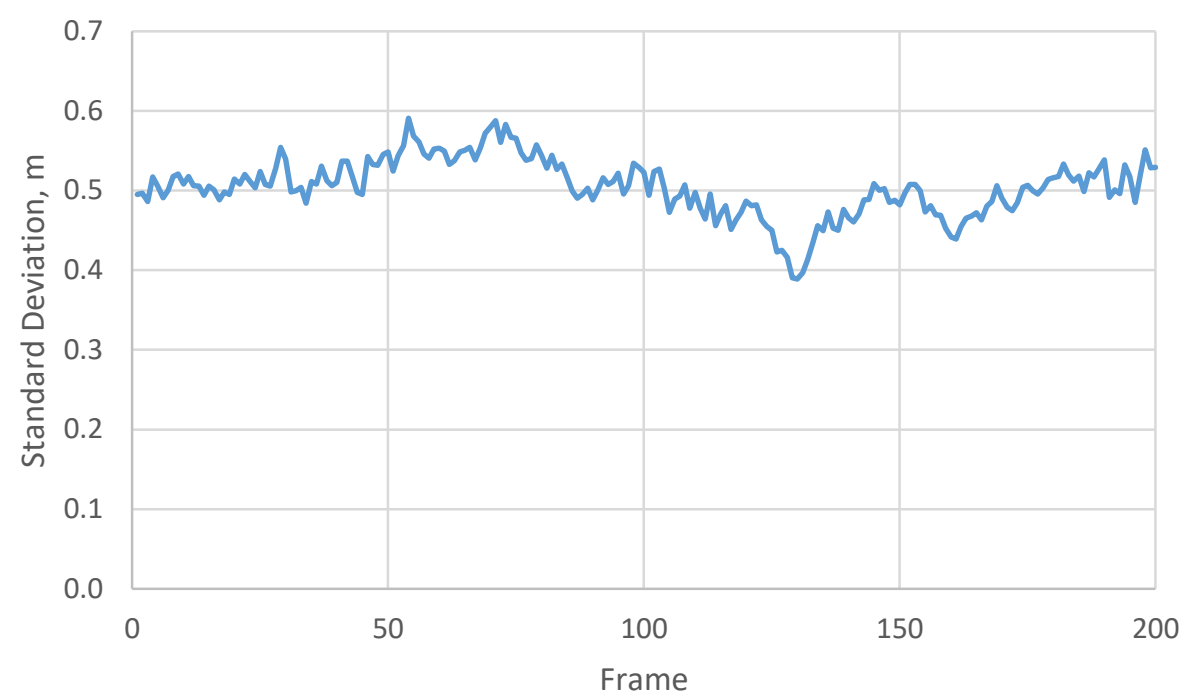

Fig. 9 The standard deviation of the point cloud representing the static obstacle.

Meanwhile, the mean absolute error and the standard deviation of the obstacle point cloud representing the moving obstacle at different time steps are presented in Fig. 10 and Fig. 11 respectively. Spikes in error are present towards the start of the experiment and at its end. The spikes at the start of the experiment could be attributed to the time it takes for the states of the particle filter to converge to their correct values. On the other hand, the spikes at the end of the experiment could be attributed to the fact that the obstacle is exiting the field of view of the camera and hence the uncertainty increases.

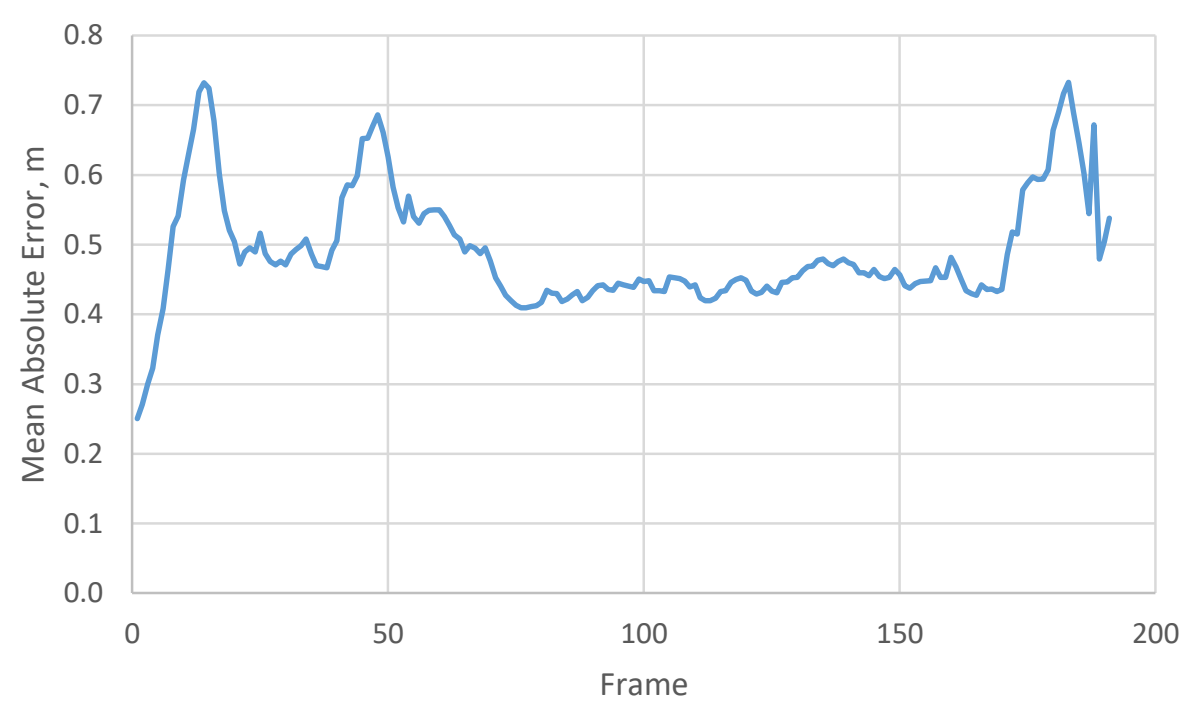

Fig. 10 The mean absolute error of the point cloud representing the moving obstacle. 


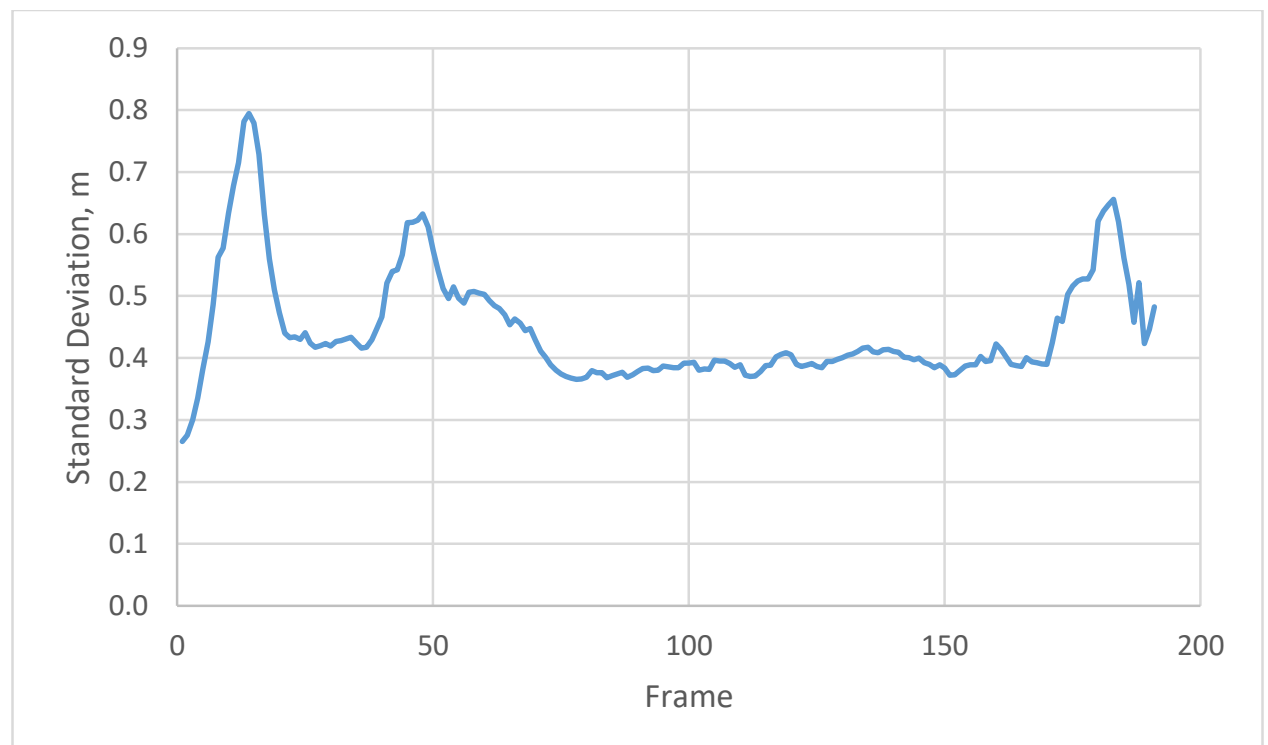

Fig. 11 The standard deviation of the point cloud representing the moving obstacle.

\section{Conclusion}

In this paper, a particle filter that tracks obstacle point clouds over time has been presented. The proposed algorithm can collect data from different viewpoints to eventually reconstruct entire obstacles in 3D. Experiments demonstrate that the developed algorithm successfully detects and tracks obstacles with mean absolute errors within $0.8 \mathrm{~m}$ and standard deviations within $0.8 \mathrm{~m}$. Unfortunately, due to a down-sampling procedure at every time step, the accuracy does not improve over time. Future work will modify the voxel grid to take note of the total number of points within each cell so that the distribution is not lost and in turn this could be used to improve the accuracy.

\section{References}

[1] G. Li, S. P. Baker, M. W. Lamb, J. G. Grabowski and G. W. Rebok, "Human factors in aviation crashes involving older pilots," Aviation, space, and environmental medicine, vol. 73, no. 2, pp. 134-138, 2002.

[2] NTSB, "Aviation Accident Factual Report DCA17CA195A," 12 September 2017. [Online]. Available: https://app.ntsb.gov/pdfgenerator/ReportGeneratorFile.ashx?EventID=20170913X72254\&AKey=1\&RType=HTML\&ITy pe $=$ CA.

[3] NTSB, “Aviation Accident Preliminary Report DCA18LA024A," 27 November 2017. [Online]. Available: https://app.ntsb.gov/pdfgenerator/ReportGeneratorFile.ashx?EventID=20171128X93434\&AKey=1\&RType=HTML\&ITy pe $=$ LA.

[4] NTSB, “Aviation Accident Final Report DCA17CA147A,” 30 June $2017 . \quad$ [Online]. Available: https://app.ntsb.gov/pdfgenerator/ReportGeneratorFile.ashx?EventID=20170703X71412\&AKey=1\&RType=HTML\&ITy pe $=$ CA.

[5] “All Condition Operations and Innovative Cockpit Infrastructure,” [Online]. Available: http://www.alicia-project.eu/CMS/. [Accessed 30 May 2018].

[6] A. D. Andre, "Information requirements for low-visibility taxi operations: What pilots say.," in Proceedings of the Eighth International Symposium on Aviation Psychology, 1995.

[7] J. Gauci and D. Zammit-Mangion, "Obstacle Detection Around Aircraft on Ramps and Taxiways Through the Use of Computer Vision," in AIAA Guidance, Navigation, and Control Conference, Chicago, Illinois, 2009.

[8] J. Shi, "Obstacle detection using thermal imaging sensors for large passenger airplane," Cranfield University, 2012.

[9] K. Theuma, D. Zammit-Mangion, J. Gauci, K. Chircop and N. Riviere, "Surface reconstruction through image postprocessing for ground obstacle detection in the airfield," in International IR Target and Background Modeling \& Simulation Workshop, Banyuls-sur-mer, France, 2018.

[10] P. D. Moral, "Nonlinear filtering: Interacting particle resolution," Comptes Rendus de l'Académie des Sciences - Series I Mathematics, vol. 325, no. 6, pp. 653-658, 1997. 
[11] H. Hirschmuller, "Stereo Processing by Semiglobal Matching and Mutual Information," IEEE Transactions on Pattern Analysis and Machine Intelligence, vol. 30, no. 2, pp. 328-341, 2007.

[12] S. Hadjitheophanous, C. Ttofis, A. S. Georghiades and T. Theocharides, "Towards hardware stereoscopic 3D reconstruction a real-time FPGA computation of the disparity map," in Design, Automation \& Test in Europe Conference \& Exhibition (DATE), Dresden, Germany, 2010.

[13] H. Cho, Y.-W. Seo, B. V. Kumar and R. R. Rajkumar, "A multi-sensor fusion system for moving object detection and tracking in urban driving environments," in IEEE International Conference on Robotics and Automation (ICRA), Hong Kong, China, 2014.

[14] M. Schutz, N. Appenrodt, J. Dickmann and K. Dietmayer, "Occupancy Grid Map-Based Extended Object Tracking," in IEEE Intelligent Vehicles Symposium (IV), Dearborn, Michigan, USA, 2014.

[15] J. Artieda, J. M. Sebastian, P. Campoy, J. F. Correa, I. F. Mondragón, C. Martínez and M. Olivares, "Visual 3-D SLAM from UAVs," Journal of Intelligent and Robotic Systems, vol. 55, no. 4-5, pp. 299-321, 2009.

[16] R. Ueda, "pcl::tracking,", [Online].

http://www.pointclouds.org/assets/files/presentations/ueda_internship_presentation.pdf. [Accessed 31 May 2018].

[17] J. Sklansky, "Finding the convex hull of a simple polygon," Pattern Recognition Letters, vol. 1, no. 2, pp. 79-83, 1982.

[18] "Blender," [Online]. Available: https://www.blender.org/. [Accessed 26 November 2018].

[19] “CloudCompare,” [Online]. Available: https://www.danielgm.net/cc/. [Accessed 26 November 2018]. 